\title{
CliniMACS CD34 Reagent System
}

National Cancer Institute

\section{Source}

National Cancer Institute. CliniMACS CD34 Reagent System. NCI Thesaurus. Code C148264.

A proprietary flow cytometry system that uses anti-CD34 antibodies to isolate CD34+ cells from apheresis specimens. 